\title{
Dispersion relation analysis of turbulent magnetic field fluctuations in fast solar wind
}

\author{
C. Perschke ${ }^{1,2}$, Y. Narita ${ }^{2,3}$, S. P. Gary ${ }^{4}$, U. Motschmann ${ }^{1,5}$, and K.-H. Glassmeier ${ }^{2,6}$ \\ ${ }^{1}$ Institut für Theoretische Physik, Technische Universität Braunschweig, Mendelssohnstr. 3, 38106 Braunschweig, Germany \\ ${ }^{2}$ Institut für Geophysik und extraterrestrische Physik, Technische Universität Braunschweig, Mendelssohnstr. 3, 38106 \\ Braunschweig, Germany \\ ${ }^{3}$ Space Research Institute, Austrian Academy of Sciences, Schmiedlstr. 6, 8042 Graz, Austria \\ ${ }^{4}$ Space Science Institute, Boulder, Colorado 80301, USA \\ ${ }^{5}$ Deutsches Zentrum für Luft- und Raumfahrt, Institut für Planetenforschung, Rutherfordstr. 2, 12489 Berlin, Germany \\ ${ }^{6}$ Max-Planck-Institut für Sonnensystemforschung, Max-Planck-Str. 2, 37191 Katlenburg-Lindau, Germany
}

Correspondence to: C. Perschke (c.perschke@tu-bs.de)

Received: 22 July 2013 - Revised: 16 October 2013 - Accepted: 17 October 2013 - Published: 15 November 2013

\begin{abstract}
Physical processes of the energy transport in solar wind turbulence are a subject of intense studies, and different ideas exist to explain them. This manuscript describes the investigation of dispersion properties in short-wavelength magnetic turbulence during a rare high-speed solar wind event with a flow velocity of about $700 \mathrm{~km} \mathrm{~s}^{-1}$ using magnetic field and ion data from the Cluster spacecraft. Using the multipoint resonator technique, the dispersion relations (i.e., frequency versus wave-number values in the solar wind frame) of turbulent magnetic fluctuations with wave numbers near the inverse ion inertial length are determined. Three major results are shown: (1) the wave vectors are uniformly quasiperpendicular to the mean magnetic field; (2) the fluctuations show a broad range of frequencies at wavelengths around the ion inertial length; and (3) the direction of propagation at the observed wavelengths is predominantly in the sunward direction. These results suggest the existence of high-frequency dispersion relations partly associated with normal modes on small scales. Therefore nonlinear energy cascade processes seem to be acting that are not described by wave-wave interactions.
\end{abstract}

Keywords. Space plasma physics (transport processes; turbulence; wave-particle interactions)

\section{Introduction}

Solar wind is often believed to be in a fully developed turbulent state. Yet, energy transport across different scales (or energy cascade) in collisionless plasmas is one of the unsolved problems, and different scenarios exist. The fact that fluctuation amplitudes are small compared to the background magnetic field leads us to hypothesize that the weak turbulence approximation may be valid in solar wind turbulence. In this approach various linear modes are considered to be nearly independent components of the turbulent fluctuations and their interactions to be weak so that many collisions of wave packets are needed to reach higher frequencies and wave numbers. Our concern here is short-wavelength turbulence, that is, fluctuations with wavelengths of the order of less than the proton inertial length. Two such linear modes are particularly relevant: kinetic Alfvén waves (KAWs) (Sahraoui et al., 2012) and oblique magnetosonic/whistler (MS/WHS) waves (Gary and Smith, 2009; Gary et al., 2012). Also, ion cyclotron waves at quasi-parallel propagation (He et al., 2012) and ion Bernstein (IB) waves at quasi-perpendicular propagation (Verscharen et al., 2012; Podesta, 2012) are likely to exist in short-wavelength plasma turbulence.

One of the main problems in wave analysis of solar wind turbulence is the Doppler-shifted frequencies in the spacecraft frame $\omega_{\mathrm{sc}}=\omega_{\mathrm{re}}+\boldsymbol{k} \cdot \boldsymbol{V}_{\mathrm{SW}}$, where subscripts "sc" and "re" denote the spacecraft and the solar wind rest frame, respectively, and $\boldsymbol{k}$ and $\boldsymbol{V}_{\mathrm{SW}}$ denote the wave vector and the 
solar wind velocity. While single-spacecraft data analyses often require Taylor's hypothesis (Taylor, 1938) neglecting frequencies in the rest frame $\omega_{\text {re }}$, the dispersion relation analysis by multi-spacecraft data is a powerful method of separating spatial and temporal variations. Multi-spacecraft missions like Cluster (Escoubet et al., 2001) provide the opportunity for such a task, and can determine the wave vector $\boldsymbol{k}$ directly and thereby determine the Doppler shift $\boldsymbol{k} \cdot \boldsymbol{V}_{\mathrm{SW}}$ unambiguously.

The fluctuation properties are different between the slow and the fast solar wind at $1 \mathrm{AU}$. While slow-wind turbulence shows characteristics of counter-propagating waves in the plasma rest frame with respect to the Sun-to-Earth direction, the fast wind is dominated by anti-sunward propagating waves on large scales (Marsch and Tu, 1990a). Dasso et al. (2005) found a slab fluctuation geometry associated with wave vectors quasi-parallel to the background magnetic field in fast solar wind turbulence and a two-dimensional geometry associated with perpendicular wave vectors in the slow solar wind. As weak turbulence conditions seem to be fulfilled at sufficiently short wavelengths in the solar wind, we hereby raise the question if it is possible to describe the highspeed solar wind turbulence as an interacting set of linear wave modes.

Studies about mode identification in solar wind turbulence have been done for a long time by using the magnetic and electric field spectrum (Leamon et al., 1998; Bale et al., 2005; Salem et al., 2012) as well as investigations of magnetic variance anisotropy (TenBarge et al., 2012) and suggest that KAWs are the main constituent of short-wavelength solar wind turbulence. Sahraoui et al. (2010a) and Roberts et al. (2013) confirm these observations, since they identify only KAWs at frequencies lower than the gyrofrequency of the protons $\left(\omega<\Omega_{\mathrm{p}}\right)$ using the k-filtering (or wave telescope) technique (Pinçon et al., 1991; Motschmann et al., 1996; Glassmeier et al., 2001). In contrast, the analysis done by Narita et al. (2011a) using the multi-point signal resonator technique, MSR (Narita et al., 2011b), showed highfrequency $\left(\omega>\Omega_{\mathrm{p}}\right)$ fluctuations in weak agreement with MS/WHS modes but without evidence of KAWs at scales around the inertial length of the ions.

In this paper we study a case of high-speed solar wind turbulence at ion kinetic scales using the MSR technique, which is very useful for dispersion relation analysis due to the enhanced signal-to-noise ratio compared to the wave telescope spectrum. Our analysis shows strong evidence of quasi-perpendicular wave vectors with respect to the background magnetic field, a broad range of frequencies $(0<\omega \lesssim$ $8 \Omega_{\mathrm{p}}$ ) over $0<k V_{\mathrm{A}} / \Omega_{\mathrm{p}}<3$, where $V_{\mathrm{A}}$ denotes the Alfvén velocity, without a clear $\omega(k)$ dispersion relation, and weak evidence of sunward propagating fluctuations at wavelengths smaller than the ion inertial length of the protons is found.

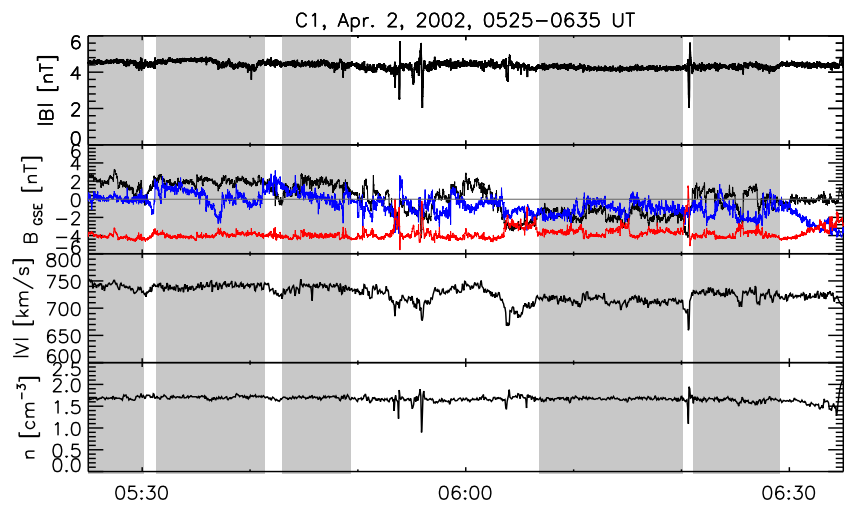

Fig. 1. Time-series data of the fast solar wind event measured by Cluster-1: magnetic field magnitude $|B|$ and components in GSE coordinates: $B_{x}$ (black), $B_{y}$ (blue), and $B_{z}$ (red) in the two top panels, ion bulk velocity $\left|\boldsymbol{V}_{\mathrm{SW}}\right|$ in the middle and ion number density $n_{i}$ in the bottom panel. The gray shaded time intervals are used for the analysis.

\section{Data selection and analysis}

On 2 April 2002, 05:25-06:35 UT, the Cluster satellites encountered a high-speed solar wind event in front of the Earth while forming a nearly regular tetrahedron with separation of about $100 \mathrm{~km}$ and $Q_{\mathrm{GM}}$ in the range of 2.62 to 2.72 . The index $Q_{\mathrm{GM}}$ characterizes the satellite spatial configuration: the regular tetrahedron geometry is represented by $Q_{\mathrm{GM}}=3$, a plane where all satellites lie by $Q_{\mathrm{GM}}=2$, and a collinear geometry by $Q_{\mathrm{GM}}=1$, respectively (vom Stein et al., 1992; Robert et al., 1998). We use time-series data registered by the fluxgate magnetometer (FGM) (Balogh et al., 2001) on board Cluster and the Cluster ion spectrometry experiment (CIS) (Rème et al., 2001) at sampling rates of $22 \mathrm{~Hz}$ and $4 \mathrm{~s}$, respectively. We split them into 5 sub-intervals with lengths of 5-13 min (Fig. 1), so that all these sub-intervals exhibit an almost constant local flow velocity and magnetic field with magnitude variation about $0.1 \mathrm{nT}$ and directional variation about $10^{\circ}$ with about $18^{\circ}$ for the last interval. Thus, we conclude that the data fulfill the required analysis assumptions of a stationary and homogeneous situation. The averaged magnetic field magnitude $B_{0}$ over the sub-intervals is $4.3 \mathrm{nT}$; the flow velocity $V_{\mathrm{SW}}$ is between 714 and $740 \mathrm{~km} \mathrm{~s}^{-1}$, the ion number density $n$ about $1.7 \mathrm{~cm}^{-3}$. The plasma parameter for protons, $\beta_{\mathrm{p}}$, is about 2.3; the ratio of the ion temperatures perpendicular and parallel to the magnetic field $T_{\perp} / T_{\|}$is 0.9 as well as $T_{\mathrm{p}} / T_{\mathrm{e}} \approx 3.4$, the ratio of the total ion and electron temperature. Table 1 presents the parameters for each interval. We exclude foreshock activities in the data by checking the ion distributions from CIS.

The analysis procedure is as follows. First, we transform the magnetic field data from the GSE (geocentric solar ecliptic) into the mean-field-aligned (MFA) coordinate system, where $z$ is parallel to the local mean magnetic field $\boldsymbol{B}_{0}$. The 
Table 1. Solar wind parameters of the five selected intervals. Electron temperatures were measured by the electron plasma instrument, PEACE (Johnstone et al., 1997).

\begin{tabular}{ccccccccc}
\hline Start [UT] & End [UT] & $\boldsymbol{B}_{0}[\mathrm{nT}]$ & $V_{\mathrm{SW}}[\mathrm{km}]$ & $\beta_{\mathrm{p}}$ & $T_{\perp} / T_{\|}$ & $T_{\mathrm{p}} / T_{\mathrm{e}}$ & $\Omega_{\mathrm{p}}\left[\mathrm{rad} \mathrm{s}^{-1}\right]$ & $V_{\mathrm{A}} / \Omega_{\mathrm{p}}\left[\mathrm{km} \mathrm{rad}^{-1}\right]$ \\
\hline 05:25:00 & $05: 30: 08$ & 4.41 & 740 & 2.13 & 0.80 & 3.2 & 0.41 & 180.0 \\
05:31:18 & $05: 41: 19$ & 4.37 & 740 & 2.23 & 0.89 & 3.4 & 0.41 & 178.7 \\
05:42:59 & $05: 49: 19$ & 4.30 & 737 & 2.42 & 0.89 & 3.4 & 0.41 & 179.0 \\
06:06:46 & $06: 20: 05$ & 4.10 & 714 & 2.65 & 0.79 & 3.5 & 0.39 & 180.6 \\
06:21:06 & $06: 29: 07$ & 4.06 & 725 & 2.55 & 1.04 & 3.5 & 0.39 & 182.8 \\
\hline
\end{tabular}

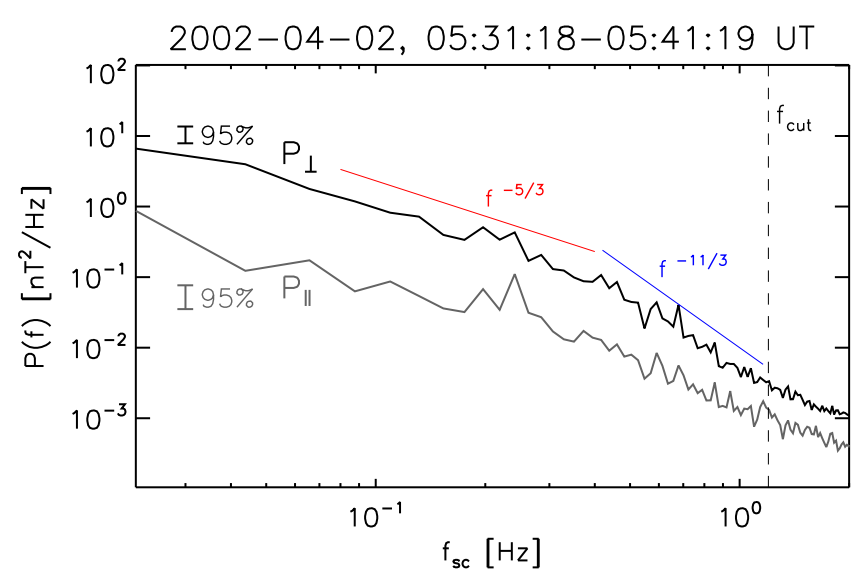

Fig. 2. Power spectra of the magnetic fluctuations parallel and perpendicular to $\boldsymbol{B}_{0}$ of the second interval with spectral break around $f_{\mathrm{sc}} \approx 0.4 \mathrm{~Hz}$. The peak at $0.25 \mathrm{~Hz}$ is spacecraft spin frequency.

$y$ axis results from $\boldsymbol{B}_{0} \times \boldsymbol{V}_{\mathrm{SW}}$, and the $x$ axis completes the right-hand system.

Second, the power spectrum in the spacecraft frame of the magnetic fluctuations for each interval is estimated by fast Fourier transform (FFT) with $N$ non-overlapping (i.e., independent) windows containing 1024 data points. Figure 2 displays representatively the power spectra of parallel, $P_{\| \mid}=P_{z}$, and perpendicular fluctuations, $P_{\perp}=\sqrt{P_{x}^{2}+P_{y}^{2}}$, with respect to the mean magnetic field $\boldsymbol{B}_{0}$ of the second interval. It represents a typical solar wind spectrum in that the perpendicular fluctuations dominate and in that a spectral break appears around $0.4 \mathrm{~Hz}$ separating it into two distinct spectral curves (Leamon et al., 1998) with spectral indexes of about $-5 / 3$ and $-11 / 3$, respectively. The frequency around the spectral break is comparable to the frequency of the Dopplershifted ion gyroradius, $f_{\rho}=V_{\mathrm{SW}} / 2 \pi \rho=0.47 \mathrm{~Hz}$, where $\rho$ denotes the ion gyroradius. The maximum frequency for the wave vector analysis is $f_{\text {cut }}=1.2 \mathrm{~Hz}$, where the FGM measurements reach the noise level indicated by the flattening at the end of the spectrum. The peak at $0.25 \mathrm{~Hz}$ occurs due to the spacecraft spin. The $95 \%$ confidence intervals are evaluated for $2 \times 4 \times N$ and $4 \times N$ degrees of freedom, representing the two perpendicular components and one parallel compo- nent, the $N$ independent windows, and measurements of the four satellites.

Third, the satellite separation and configuration as well as the the flow velocity limit the accessible wave-number and frequency range. The maximum wave number corresponds to $k_{\max }=0.022 \mathrm{rad} \mathrm{km}^{-1}$ (Glassmeier et al., 2001). The minimal wave number is set to $k_{\min }=k_{\max } / 10$, suggested by numerical studies of Sahraoui et al. (2010b), such that the relative error of the determined wave vector is smaller than $10 \%$. Applying the analysis to spacecraft frequencies lower than $k_{\max } \cdot V_{\mathrm{SW}} / 2 \pi=2.5 \mathrm{~Hz}$ avoids spatio-temporal aliasing what is met by the limitation due to the noise frequency $f_{\text {cut }}$. Frequencies around the spin frequencies are excluded from the wave vector analysis, too.

The adaptive MSR technique estimates the wave power $P(\omega, \boldsymbol{k})$ at a certain frequency $\omega$ and wave vector $\boldsymbol{k}$ using a minimum variance projection weighted by the orthonormal condition between noise and signal eigenvectors of crossspectral density of the magnetic field data. Thus, the MSR technique in combination with the FFT settings enables us to determine the wave power $P\left(\omega_{\mathrm{sc}}, \boldsymbol{k}\right)$ at various wave vectors for wave frequencies $\omega_{\mathrm{sc}} \leq 2 \pi f_{\text {cut }}$ with a sampling step $\delta \omega=$ $0.135 \mathrm{rad} \mathrm{s}^{-1}$ assuming coherent, long-time existing, plane wave structures. Here, the spherical wave vector domain is discretized into a $100 \times 60 \times 70$ grid in radial, azimuthal, and polar directions. The sampling steps are $\delta k=k_{\max } / 100$, $\delta \varphi=6^{\circ}$ and $\delta \theta=1^{\circ}$ close to 0,90 , and $180^{\circ}$, otherwise $5^{\circ}$. A set of frequency and wave vector is obtained at which waves exist clearly if a value of $P\left(\omega_{\mathrm{sc}}, \boldsymbol{k}\right)$ is a local maximum at the wave vector $\boldsymbol{k}$ for a chosen wave frequency $\omega_{\mathrm{sc}}$, graphically represented in Fig. 3 as the projection of the spectrum into the wave-number planes (reduced power spectrum).

Finally, since we are interested in the dispersion relations in the plasma rest frame, we correct for the Doppler shift and plot the $\left(\omega_{\mathrm{re}}, k\right)$ combinations in Fig. 4 . The wave frequency $\omega_{\text {re }}$ is normalized to the proton gyrofrequency $\Omega_{\mathrm{p}}=0.41 \mathrm{rad} \mathrm{s}^{-1}$ and the wave number to the proton inertial length $V_{\mathrm{A}} / \Omega_{\mathrm{p}}=178 \mathrm{~km}$, where $V_{\mathrm{A}}=72 \mathrm{~km} \mathrm{~s}^{-1}$ is the Alfvén velocity. The error of the estimated frequencies in the rest frame is that of the Doppler shift (Narita et al., 2003). Hence the relative error of the frequencies is $\left|\delta \omega_{\text {re }} / \omega_{\text {re }}\right| \leq$ $|\delta k / k|+|\delta V / V| \leq 0.12$. The standard deviations of the ion velocity data are used for this estimation. 


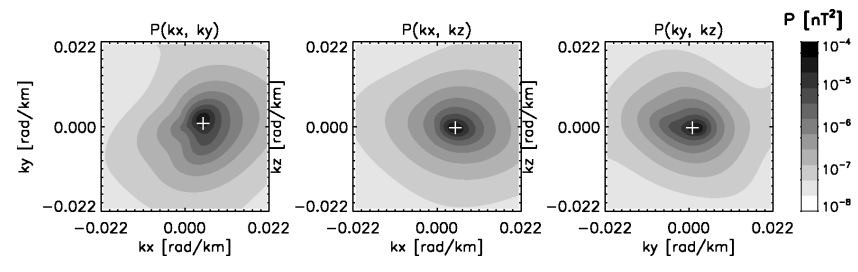

Fig. 3. Example of a reduced three-dimensional power spectrum from the second interval $(05: 31-05: 41 \mathrm{UT})$ at $\omega_{\mathrm{sc}}=3.44 \mathrm{rad} \mathrm{s}^{-1}$. The white crosses indicate the maximum of the power spectrum.

\section{Results}

The results of the analysis are presented in Fig. 4. The quasiperpendicular wave vector orientation with respect to the mean magnetic field at various wave numbers across the ion inertial length is clearly visible in the upper panel showing the angle $\theta_{\mathrm{kB}}$ between the wave vectors and the background magnetic field. The average of all measured angles is $\left\langle\theta_{\mathrm{kB}}\right\rangle=92^{\circ}$ with a standard deviation of $7^{\circ}$. On the other hand, the observed points in the lower panel show an increase in frequency spreading for increasing wave numbers up to an order of magnitude, suggesting, as in Narita et al. (2011a), that identification of a specific normal mode may be difficult. In addition to this earlier work, we use numerical solutions of the full kinetic linear dispersion equation (Gary, 1993) and obtained frequency versus wave-number plots for several different modes as plotted in Fig. 4. From the comparison, several conclusions can be drawn.

First, consider the solid lines, which represent the magnetosonic waves at $\omega_{\mathrm{re}}<\Omega_{\mathrm{p}}$ and the lightly damped regimes of the lowest frequency proton Bernstein (PB) harmonic modes at quasi-perpendicular propagation (in this case $\theta=80^{\circ}$ ). Over the frequency range $\Omega_{\mathrm{p}} \leq \omega_{\text {re }} \leq 5 \Omega_{\mathrm{p}}$, some observed dispersion relations show weak agreement with these normal modes.

Second, the short dashed line represents the MS/WHS mode at $\theta=60^{\circ}$ where the PB modes are suppressed. Here, this obliquely propagating mode is a plausible representation of the wave properties. Its distinctly positive dispersion $\left(\partial^{2} \omega_{\text {re }} / \partial k^{2}>0\right)$ is a relatively weak function of $\theta$ and possesses features similar to the observations. Narita et al. (2011a) obtained a similar agreement using a cold plasma representation for MS/WHS dispersion.

Third, the long dashed line represents the weakly damped kinetic Alfvén mode propagating at $80^{\circ}$ relative to the background magnetic field. The dispersion of this mode is proportional to $k_{\|}$so that, given the strongly oblique propagation of each mode observed here, this curve represents an approximate upper bound for any correlation with the observations. Thus, as in Narita et al. (2011a), the substantial majority of points on the frequency-wave-number distribution lie closer to the predicted MS/WHS dispersion curve than to the predicted KAWs.

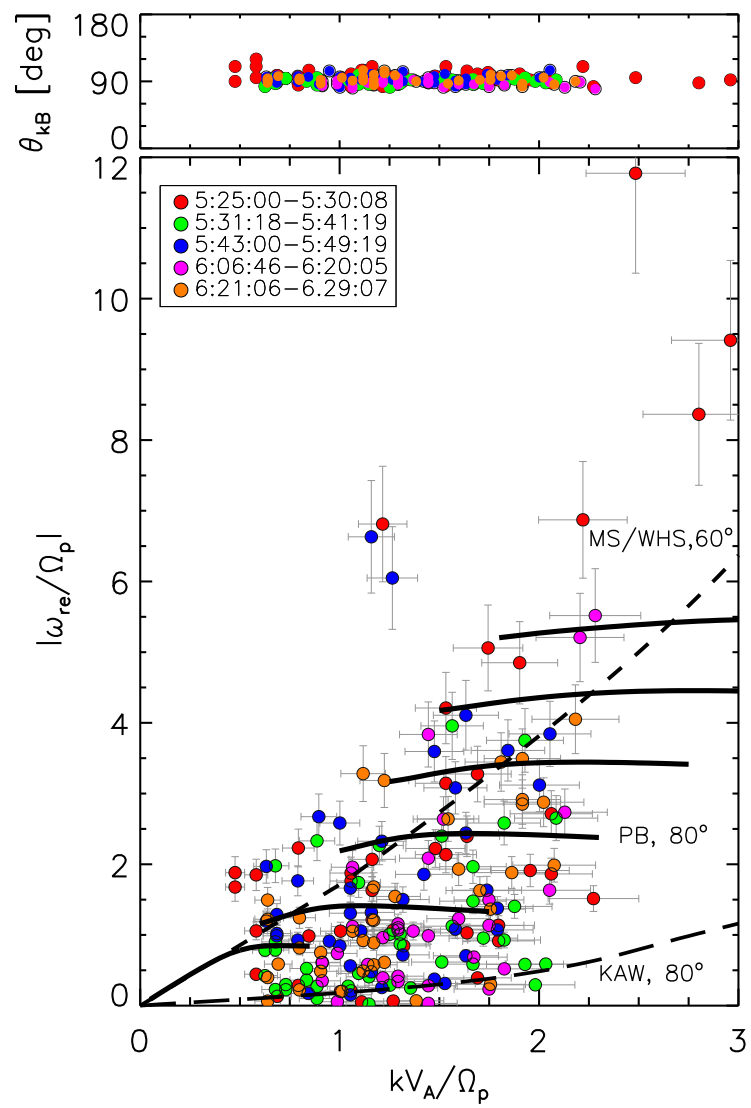

Fig. 4. Frequency-wave-number diagram. The angle $\theta_{\mathrm{kB}}$ between determined wave vectors and the background magnetic field is shown in the top panel indicating the quasi-perpendicular wave vector characteristic. The lower panel shows the distribution of the observed dispersion relations as well as linear theory dispersion relations for the magnetosonic/whistler (MS/WHS) and proton Bernstein (PB, solid) modes at $\theta=80^{\circ}$, the MS/WHS waves at $\theta=60^{\circ}$ (dashed line), and the kinetic Alfvén waves (KAWs, long dashed line) at $\theta=80^{\circ}$. Error bars are plotted in light gray.

The analysis also provides information about the direction of wave propagation in the plasma rest frame. A negative value of $\omega_{\text {re }}$ indicates sunward direction of the wave vector $\boldsymbol{k}$, and a positive $\omega_{\text {re }}$ represents anti-sunward propagation. To determine the sign of dominant energy transport direction, we calculated the wave power ratio $\left(P_{+}-P_{-}\right) /\left(P_{+}+P_{-}\right)$, where $P_{+}$denotes the power of waves with positive $\omega_{\text {re }}$ and $P_{-}$for negative values, respectively, binned in the wavenumber domain. The result is displayed in Fig. 5, where the value -1 is associated with dominant sunward propagation and +1 with a dominant anti-sunward one. The distribution exhibits two populations: the first one around $k V_{\mathrm{A}} / \Omega_{\mathrm{p}} \lesssim 1$ indicates an energy balance in counter-propagating waves; the second population at $k V_{\mathrm{A}} / \Omega_{\mathrm{p}} \gtrsim 1$ is dominated by sunward propagating waves. The error bars refer to the width of the binning intervals and the variance of the determined 


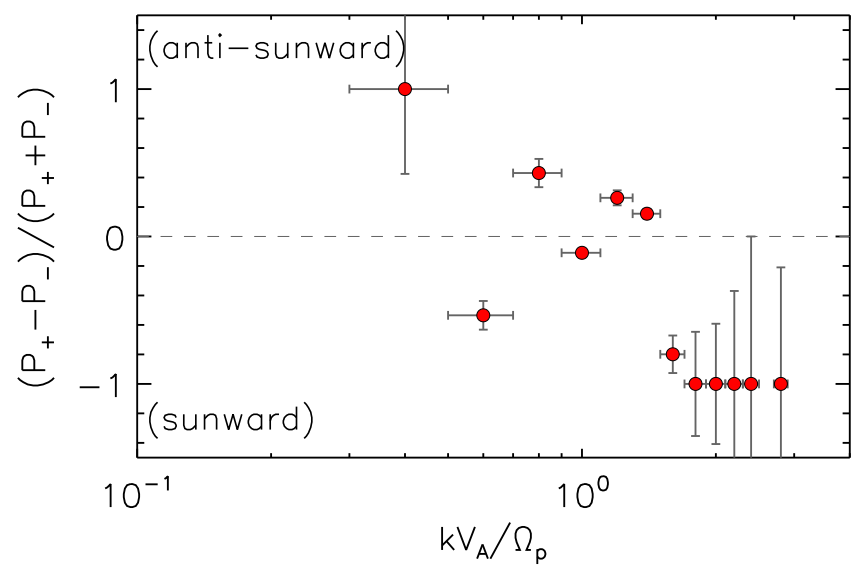

Fig. 5. Power ratio between sunward and anti-sunward propagating waves.

power ratio values, respectively. The average number of data sets in one binning interval is about 15 .

\section{Discussion and conclusion}

Using magnetic field and ion measurements from the Cluster spacecraft during an interval of high-speed flow in the solar wind, we have used the MSR technique to analyze the plasma frame frequency versus wave-number dispersion relation for turbulent magnetic fluctuations. We find three major results for fluctuations with $k V_{\mathrm{A}} / \Omega_{\mathrm{p}} \sim 1$. First, the wave vectors of almost all the fluctuations are quasi-perpendicular to the local average magnetic field. Second, the plasma frame frequencies at a given wave number range from nearly zero to several times $\Omega_{p}$ with weak agreement with proton Bernstein and magnetosonic/whistler dispersion relations. Third, the direction of propagation in the plasma rest frame at relatively short wavelengths is predominantly in the sunward direction.

The quasi-perpendicular character of the wave vectors is consistent with previous solar wind measurements (Sahraoui et al., 2010b; Narita et al., 2011a) and with computer simulations of short-wavelength turbulence (Howes et al., 2011; Chang et al., 2011) and confirms recent studies by Chen et al. (2012), who determined the formation of a two-dimensional structure as a transition into small-scale fluctuations in the fast solar wind.

The observed dispersion relations show no clear evidence of normal modes, but the existence and dominance of highfrequency waves confirm the observations from Narita et al. (2011a) at lower flow velocities of about $400 \mathrm{~km} \mathrm{~s}^{-1}$. Figure 4 shows a very narrow distribution of propagation angles of about $90^{\circ}$ but a very broad distribution of plasma frame frequencies for a particular choice of wave number. It covers frequency and wave-number ranges of the MS/WHS and PB modes and those of the KAWs, but it does not show the narrow one-to-one correspondence between frequency and wave number predicted by linear theory. The absence of such a relationship suggests that nonlinear processes are important here, and a pure weak turbulence treatment seems not to be justified for this event unlike other multispacecraft dispersion relation observations by Sahraoui et al. (2010a) and Roberts et al. (2013). Nonlinear effects that might contribute to broadening of the frequency spectrum include wave-particle interactions (e.g., kinetic instabilities as discussed by Kasper et al. (2002), Hellinger et al. (2006), and Bale et al. (2009)), as well as wave-wave interactions (e.g., turbulent cascades as illustrated in Fig. 9 of Svidzinski et al. (2009)). Future studies should address the effects of nonlinearities on dispersion, for example, by constructing dispersion plots for different levels of fluctuation amplitudes. In our analysis we assumed the long-time existence of coherent and plane wave structures in the fluctuations so that other structures may exist but were not identified.

The discovery of a balanced ratio between sunward and anti-sunward components and a tendency of transition into sunward-orientated ones for higher wave numbers are in contrast to the scenario of Alfvénic turbulence (Marsch and Tu, 1990a). Fluctuation properties are very different between the magnetohydrodynamic and kinetic regimes.

Acknowledgements. This work was supported by CRC 963 "Astrophysical Flow, Instabilities and Turbulence" of the German Science Foundation, Bundesministerium für Wirtschaft und Technologie and Deutsches Zentrum für Luft- und Raumfahrt under contract 50 OC 1102. C. Perschke also thanks the CIS and FGM team, for providing ion and magnetic field data.

Topical Editor L. Blomberg thanks S. Bale and one anonymous referee for their help in evaluating this paper.

\section{References}

Bale, S. D., Kellogg, P. J., Mozer, F. S., Horbury, T. S., and Reme, H.: Measurement of the electric fluctuation spectrum of magnetohydrodynamic turbulence, Phys. Rev. Lett., 94, 215002, doi:10.1103/PhysRevLett.94.215002, 2005.

Bale, S. D., Kasper, J. C., Howes, G. G., Quataert, E., Salem, C., and Sundkvist, D.: Magnetic fluctuation power near proton temperature anisotropy instability thresholds in the solar wind, Phys. Rev. Lett., 103, 211101, doi:10.1103/PhysRevLett.103.211101, 2009.

Balogh, A., Carr, C. M., Acuña, M. H., Dunlop, M. W., Beek, T. J., Brown, P., Fornacon, K.-H., Georgescu, E., Glassmeier, K.H., Harris, J., Musmann, G., Oddy, T., and Schwingenschuh, K.: The Cluster Magnetic Field Investigation: overview of in-flight performance and initial results, Ann. Geophys., 19, 1207-1217, doi:10.5194/angeo-19-1207-2001, 2001.

Chang, O., Gary, S. P., and Wang, J.: Whistler turbulence forward cascade: Three-dimensional particle-in-cell simulations, Geophys. Res. Lett., 38, L22102, doi:10.1029/2011GL049827, 2011. 
Chen, C. H., Mallet, A., Schekochihin, A. A. S., Horbury, T. S., Wicks, R. T., and Bale, S. D.: Three-dimensional structure solar wind turbulence, Astrophys. J., 758, 120, doi:10.1088/0004637X/758/2/120, 2012.

Dasso, S., Milano, L. J., Matthaeus, W. H., and Smith, C. W.: Anisotropy in fast and slow solar wind fluctuations, Astrophys. J., 635, 181-184, 2005.

Escoubet, C. P., Fehringer, M., and Goldstein, M.: Introduction The Cluster mission, Ann. Geophys., 19, 1197-1200, doi:10.5194/angeo-19-1197-2001, 2001.

Gary, S. P.: Theory of Space Plasma Microinstabilities, Cambridge University Press, Cambridge, 1993.

Gary, S. P. and Smith, C. W.: Short-wavelength turbulence in the solar wind: Linear theory of whistler and kinetic Alfvén fluctuations, J. Geophys. Res., 114, A12105, doi:10.1029/2009JA014525, 2009.

Gary, S. P., Chang, O., and Wang, J.: Forward cascade of whistler turbulence: three-dimensional particle-in-cell simulations, Astrophys. J., 755, 142, doi:10.1088/0004-637X/755/2/142, 2012.

Glassmeier, K.-H., Motschmann, U., Dunlop, M., Balogh, A., Acuña, M. H., Carr, C., Musmann, G., Fornaçon, K.-H., Schweda, K., Vogt, J., Georgescu, E., and Buchert, S.: Cluster as a wave telescope - first results from the fluxgate magnetometer, Ann. Geophys., 19, 1439-1447, doi:10.5194/angeo-19-14392001, 2001.

He, J., Tu, C., Marsch, E., and Yao, S.: Do oblique Alfvén/ioncyclotron or fast-mode/whistler waves dominate the dissipation of solar wind turbulence near the proton inertial length?, Astrophys. J., 745, doi:10.1088/2041-8205/745/1/L8, 2012.

Hellinger, P., Trávniček, P., Kasper,, J. C., and Lazarus, A. J.: Solar wind proton temperature anisotropy: Linear theory and WIND/SWE observations, Geophys. Res. Lett, 33, L09101, doi:10.1029/2006GL025925, 2006.

Howes, G. G., Tenbarge, J. M., Dorland, W., Quataert, E., Schekochihin, A. A., Numata, R., and Tatsuno, T.: Gyrokinetic simulations of solar wind turbulence from ion to electron scales, Phys. Rev. Lett., 107, 035004, doi:10.1103/PhysRevLett.107.035004, 2011.

Johnstone, A. D., Alsop, C., Burge, S., Carter, P. J., Coates, A. J., Coker, A. J., Fazakerley, A. N., Grande, M., Gowen, R. A., Gurgiolo, C., Hancock, B. K., Narheim, B., Preece, A., Sheather, P. H., Winningham, J. D., and Woodliffe, R. D.: Peace: a plasma electron and current experiment, Space Sci. Rev., 79, 351-398, 1997.

Kasper, J. C., Lazarus, A. J., and Gary, S. P.: Wind/SWE observations of firehose constraint on solar wind proton temperature anisotropy, Geophys. Res. Lett., 29, 1893, doi:10.1029/2002GL015128, 2002.

Leamon, R. J., Smith, C. W., Ness, N. F., and Wong, H. K.: Observational constraints on the dynamics of the interplanetary magnetic field dissipation range, J. Geophys. Res., 103, 4775-4787, 1998.

Marsch, E. and Tu, C.-Y.: On the radial evolution of MHD turbulence in the inner heliosphere, J. Geophys. Res., 95, 8211-8229, 1990.

Motschmann, U., Woodward, T. I., Glassmeier K.-H., Southwood, D. J., and Pinçon : Wavelength and direction filtering by magnetic measurements at satellite arrays: Generalized minimum variance analysis, J. Geophys. Res., 101, 4961-4966, 1996.
Narita, Y., Glassmeier, K.-H., Schaefer, S., Motschmann, U., Sauer, K., Dandouras, I., Fornacon, K.-H., Georgescu, E., and Reme, H.: Dispersion analysis of ULF waves in the foreshock using cluster data and the wave telescope technique, Geophys. Res. Lett., 30, doi:10.1029/2003GL017432, 2003.

Narita, Y., Gary, S. P., Saito, S., Glassmeier, K. -H., and Motschmann, U.: Dispersion relation analysis of solar wind turbulence, Geophys. Res. Lett., 38, L05101, doi:10.1029/2010GL046588, 2011a.

Narita, Y., Glassmeier, K.-H., and Motschmann, U.: Highresolution wave number spectrum using multi-point measurements in space - the Multi-point Signal Resonator (MSR) technique, Ann. Geophys., 29, 351-360, doi:10.5194/angeo-29-3512011, 2011b.

Pinçon, J. L. and Lefeuvre, F.: Local characterization of homogeneous turbulence in a space plasma from simultaneous measurements of field components at several points in space, J. Geophys. Res., 96, 1789-1802, 1991.

Podesta J. J.: The need to consider ion Bernstein modes waves as a dissipation channel of solar wind turbulence, J. Geophys. Res., 117, A07101, doi:10.1029/2012JA017770, 2012.

Rème, H., Aoustin, C., Bosqued, J. M., Dandouras, I., Lavraud, B., Sauvaud, J. A., Barthe, A., Bouyssou, J., Camus, Th., Coeur-Joly, O., Cros, A., Cuvilo, J., Ducay, F., Garbarowitz, Y., Medale, J. L., Penou, E., Perrier, H., Romefort, D., Rouzaud, J., Vallat, C., Alcaydé, D., Jacquey, C., Mazelle, C., d’Uston, C., Möbius, E., Kistler, L. M., Crocker, K., Granoff, M., Mouikis, C., Popecki, M., Vosbury, M., Klecker, B., Hovestadt, D., Kucharek, H., Kuenneth, E., Paschmann, G., Scholer, M., Sckopke, N., Seidenschwang, E., Carlson, C. W., Curtis, D. W., Ingraham, C., Lin, R. P., McFadden, J. P., Parks, G. K., Phan, T., Formisano, V., Amata, E., Bavassano-Cattaneo, M. B., Baldetti, P., Bruno, R., Chionchio, G., Di Lellis, A., Marcucci, M. F., Pallocchia, G., Korth, A., Daly, P. W., Graeve, B., Rosenbauer, H., Vasyliunas, V., McCarthy, M., Wilber, M., Eliasson, L., Lundin, R., Olsen, S., Shelley, E. G., Fuselier, S., Ghielmetti, A. G., Lennartsson, W., Escoubet, C. P., Balsiger, H., Friedel, R., Cao, J.-B., Kovrazhkin, R A., Papamastorakis, I., Pellat, R., Scudder, J., and Sonnerup, B.: First multispacecraft ion measurements in and near the Earth's magnetosphere with the identical Cluster ion spectrometry (CIS) experiment, Ann. Geophys., 19, 1303-1354, doi:10.5194/angeo19-1303-2001, 2001.

Robert, P., Roux, A., Harvey, C. C., Dunlop, M. W., Daly, P. W., and Glassmeier, K.-H.: Tetrahedron geometric factors: Analysis methods for multi-spacecraft data, 323-348, ISSI Scientific Reoprt SR-001, edited by: Paschmann, G. and Daly, P. W., International Space Science Institute, ISSI/ESA, 1998.

Roberts, O. W., Li, X., and Li, B.: Kinetic plasma turbulence in the fast solar wind measured by Cluster, Astrophys. J., 769, 58, doi:10.1088/0004-637X/769/1/58, 2013.

Sahraoui, F., Goldstein, M. L., Belmont, G., Canu, P., and Rezeau, L.: Three dimensional anisotropic $\mathrm{k}$ spectra of turbulence at subproton scales in the solar wind, Phys. Rev. Lett., 105, 131101, doi:10.1103/PhysRevLett.105.131101, 2010a.

Sahraoui, F., Belmont, G., Goldstein, M. L., and Rezeau, L.: Limitations of multispacecraft data techniques in measuring wave number spectra of space plasma turbulence, J. Geophys. Res., 115, 4206, doi:10.1029/2009JA014724, 2010 b. 
Sahraoui, F., Belmont, G., and Goldstein, M. L.: New insight into short wavelength solar wind fluctuations form Vlasov theory, Astrophys. J., 748, 100, doi:10.1088/0004-637X/748/2/100, 2012.

Salem, C. S., Howes, G. G., Sundkvist, D., Bale, S. D., Chaston, C. C., Chen, C. H. K., and Mozer, F. S.: Identification of kinetic alfvén wave turbulence in the solar wind, Astrophys. J., 745, L9, doi:10.1088/2041-8205/745/1/L9, 2012.

Svidzinski, V. A., Li, H., Rose, H. A., Albright, B. J., and Bowers, K. J.: Particle in cell simulations of fast magnetosonic wave turbulence in the ion cyclotron frequency range, Phys. Plasmas, 16, 122310, doi:10.1063/1.3274559, 2009.

Taylor, G. I.: The spectrum of turbulence, Proc. R. Soc. Lond. A, 164, 476-490, 1938.
TenBarge, J. M., Podesta, J. J., Klein, K. G., and Howes, G. G., Interpreting magnetic variance anisotropy measurements in the solar wind, Astrophys. J., 753, 107, doi:10.1088/0004637X/753/2/107, 2012.

Verscharen, D., Marsch, E., Motschmann, U., and Müller, J.: Kinetic cascade beyond magnetohydrodynamics of solar wind turbulence in two-dimensional hybrid simulations, Phys. Plasmas, 19, 022305, doi:10.1063/1.3682960, 2012.

vom Stein, R., Glassmeier, K.-H., and Dunlop, M.: A Configuration Parameter for the Cluster Satellites, Tech. Rep. 2/1992, Institut für Geophysik und Meteologie der Technischen Universiät Braunschweig, 1992. 